\title{
Charge Separation Pathways in a Highly Efficient Polymer: Fullerene Solar Cell Material
}

\author{
Arun Aby Paraecattil and Natalie Banerji* \\ Institute of Chemical Sciences and Engineering, Ecole Polytechnique Fédérale de Lausanne (EPFL), SB ISIC GR-MO, Station 6, \\ CH-1015 Lausanne, Switzerland
}

Supporting Information

ABSTRACT: PBDTTPD is one of the best conjugated polymers for solar cell applications (up to $8.5 \%$ efficiency). We have investigated the dynamics of charge generation in the blend with fullerene (PCBM) and addressed highly relevant topics such as the role of bulk heterojunction structure, fullerene excitation, and excess energy. We show that there are multiple charge separation pathways. These include electron transfer from photoexcited polymer, hole transfer from photoexcited PCBM, prompt $(<100 \mathrm{fs})$ charge generation in intimately mixed polymer:fullerene regions (which can occur from hot states), as well as slower electron and hole transfer from excitons
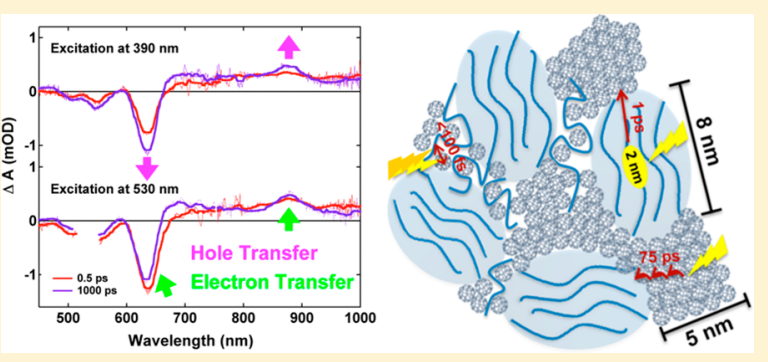
formed in pure PBDTTPD or PCBM domains (diffusion to an interface is necessary). Very interestingly, all the charge separation pathways are highly efficient. For example, the yield of long-lived carriers is not significantly affected by the excitation wavelength, although this changes the fraction of photons absorbed by PCBM and the amount of excess energy brought to the system. Overall, the favorable properties of the PBDTTPD:PCBM blend in terms of morphology and exciton delocalization allow excellent charge generation in all circumstances and strongly contribute to the high photovoltaic performance of the blend.

\section{INTRODUCTION}

Organic solar cells based on electron-donating conjugated polymers blended with electron-accepting fullerene derivatives have reached high efficiencies beyond $8 \% .^{1-3}$ In order to further enhance their performance, it is essential to deeply understand the underlying photophysical processes. Highly relevant topics that need to be addressed include the role of delocalization and exciton diffusion for efficient charge generation at the donor:acceptor interface, the role of hot neutral and charge transfer states in the formation of free carriers, and the role of the fullerene as light harvester. ${ }^{4}$ Both the nanoscale structure of the bulk heterojunction $(\mathrm{BHJ})$ and the photophysical dynamics must be considered to gain answers to those questions. Femtosecond transient absorption (TA) spectroscopy is an undeniably useful tool to study the dynamics of neutral excited states and photoinduced charges in the materials of interest. ${ }^{5-9}$ Here, we will show that this purely spectroscopic technique additionally yields detailed morphological insights. Our study leads to a comprehensive picture of how different charge generation pathways relate to $\mathrm{BHJ}$ structure.

We focus the investigation on PBDTTPD (poly (benzo[1,2$\left.b: 4,5-b^{\prime}\right]$ dithiophene-alt-thieno[3,4-c]pyrrole-4,6-dione), and its blend with PCBM $\left([6,6]\right.$-phenyl $\mathrm{C}_{60}$ butyric acid methyl ester). The polymer is among the best performing for photovoltaic applications, with up to $8.5 \%$ efficiency. ${ }^{1}$ Recent reports on morphology control, ${ }^{1,10-12}$ device design, ${ }^{13}$ charge trapping, ${ }^{14}$ long-term stability, ${ }^{15}$ quantum calculations, ${ }^{16,17}$ and spectroscopy $^{7,18-20}$ confirm high interest for the material. PBDTTPD also distinguishes itself from many other conjugated polymers through its very planar structure in both the ground and excited state (weak torsional relaxation), ${ }^{17,20}$ favoring delocalization effects. Moreover, we have previously shown that the positive PBDTTPD polaron has an exceptionally characteristic signature, ${ }^{7}$ particularly suitable for visualizing interfacial charge separation (CS) processes. Here, we present further TA results for thin films of neat and blended PBDTTPD, recorded at different excitation wavelengths $\left(\lambda_{\mathrm{ex}}\right)$, including polarizationsensitive measurements. TA studies as a function of $\lambda_{\mathrm{ex}}$ on conjugated polymers remain relatively scarce $5,6,21,22$ but are extremely useful. First, they allow evaluating the effect of excess energy on relaxation processes in neat polymers and on the generation of free charge carriers from "hot" states in the fullerene blends. ${ }^{5,21,23-25}$ Second, the relative fraction of photons absorbed by the polymer and fullerene can be controlled. Although the polymer electron donors in $\mathrm{BHJ}$ solar cells are usually considered to harvest the majority of light, photoexcitation of the acceptor followed by hole transfer can also occur, especially at short wavelengths and for strongly absorbing acceptors. ${ }^{8,19,26-31}$

\section{RESULTS AND DISCUSSION}

The molecular structure of the polymer and the steady-state absorption spectra of the used PBDTTPD and PBDTTPD:PCBM samples are shown in Figure 1, where vertical lines indicate the TA pump wavelengths. The samples

Received: October 8, 2013 


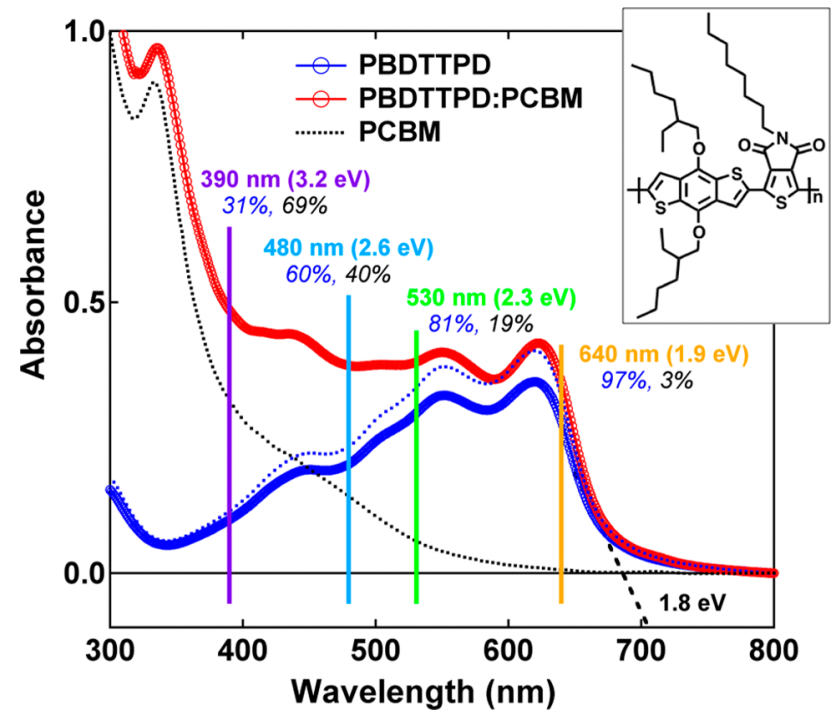

Figure 1. Steady-state absorption spectra of the used thin films of PBDTTPD and PBDTTPD:PCBM (1:2 weight ratio, cast from odichlorobenzene) are shown as blue and red lines with circular markers. Dotted spectra of the polymer and PCBM are scaled so that their sum reproduces the absorption of the PBDTTPD:PCBM sample. Vertical lines represent the TA excitation wavelengths, with the relative fraction of photons absorbed by PBDTTPD (blue) and PCBM (black) indicated in italics. (Inset) Molecular structure of PBDTTPD.

were excited with an excess energy ranging from 0.1 to $1.4 \mathrm{eV}$ compared to the $1.8 \mathrm{eV}$ polymer absorption edge. We have previously found that extremely low excitation fluence $(<4 \mu \mathrm{J} /$ $\mathrm{cm}^{2}$ ) is necessary to avoid laser-induced exciton annihilation and charge recombination artifacts for PBDTTPD-based samples. ${ }^{7}$ With an even improved experimental sensitivity, we have now carefully recorded all data below this threshold and verified that the dynamics for each measurement are fluence-independent in this regime. The flux of absorbed photons at the various excitation wavelengths was kept constant at $5.9 \times 10^{12}$ photons/ $\mathrm{cm}^{2}$ for the blend and at $4.9 \times 10^{12}$ photons $/ \mathrm{cm}^{2}$ for the neat polymer, taking into account the absorbance at $\lambda_{\mathrm{ex}}$ the fluence and the photon energy (see explanation in the Supporting Information [SI] and Table S1).
Excited-State Relaxation in Neat PBDTTPD. The TA spectra of neat PBDTTPD film upon $390 \mathrm{~nm}$ excitation are shown in Figure 2A, with the typical signatures for ground-state bleaching (GSB, 460-680 nm), stimulated emission (SE, 640$750 \mathrm{~nm}$ ), and excited-state absorption (ESA, 740-950 nm). At early times, spectral shifts occur especially in the SE region due to excited-state relaxation, as emphasized in the normalized TA spectra at the bottom of Figure 2A. We used the sum of exponential functions to globally analyze the TA dynamics at several probe wavelengths (Figures $2 \mathrm{~B}$ and S9, SI). The multiphasic fast relaxation could be reproduced with time constants of 1.6 and 18 ps. We have previously described the detailed interpretation of this multiexponential global analysis of the TA dynamics for PBDTTPD film. ${ }^{7}$ Both structural backbone relaxation and exciton hopping between chromophoric units formed by conformational breaks in the polymer chain contribute in general to such relaxation processes in conjugated polymers. ${ }^{4,32-36}$ Given the planarity and limited torsional relaxation in PBDTTPD, ${ }^{17,20}$ the second relaxation effect is predominant here. As seen in Figure 2A, there is some groundstate recovery in parallel with this fast relaxation, pointing to decay of the excited state. However, the majority of neutral excitons decay with a time constant of $250 \mathrm{ps}$, which is slightly longer than the 150 ps time constant we initially reported, ${ }^{7}$ as we used a different polymer batch and even lower pump intensity. Finally, some GSB and absorption features survive at long times, due to population of polaron and/or triplet states (the triplet yield in PBDDTPD film has been reported to be $6 \%) .{ }^{19}$

The dynamics recorded at different positions of the TA spectrum have no dependence on $\lambda_{\mathrm{ex}}$ (Figure 2B, more dynamics and outcome of global analysis are shown in Figure S9, SI). According to quantum calculations, at least two electronic transitions are present in the broad $350-700 \mathrm{~nm}$ steady-state absorption band, superposed to vibronic peaks caused by coupling to $\mathrm{C}-\mathrm{C}$ stretching modes. ${ }^{17} \mathrm{We}$ conclude that the excess energy introduced depending on $\lambda_{\mathrm{ex}}$ is dissipated by internal conversion (IC) and thermalization processes faster than the $\sim 100-150$ fs experimental time resolution. At longer times, $\lambda_{\mathrm{ex}}$ has no influence on the dynamics of exciton hopping, although such an effect might be expected since selective excitation of low energy sites prevents further downhill relaxation. ${ }^{36}$ Most probably, our quite broadband femtosecond
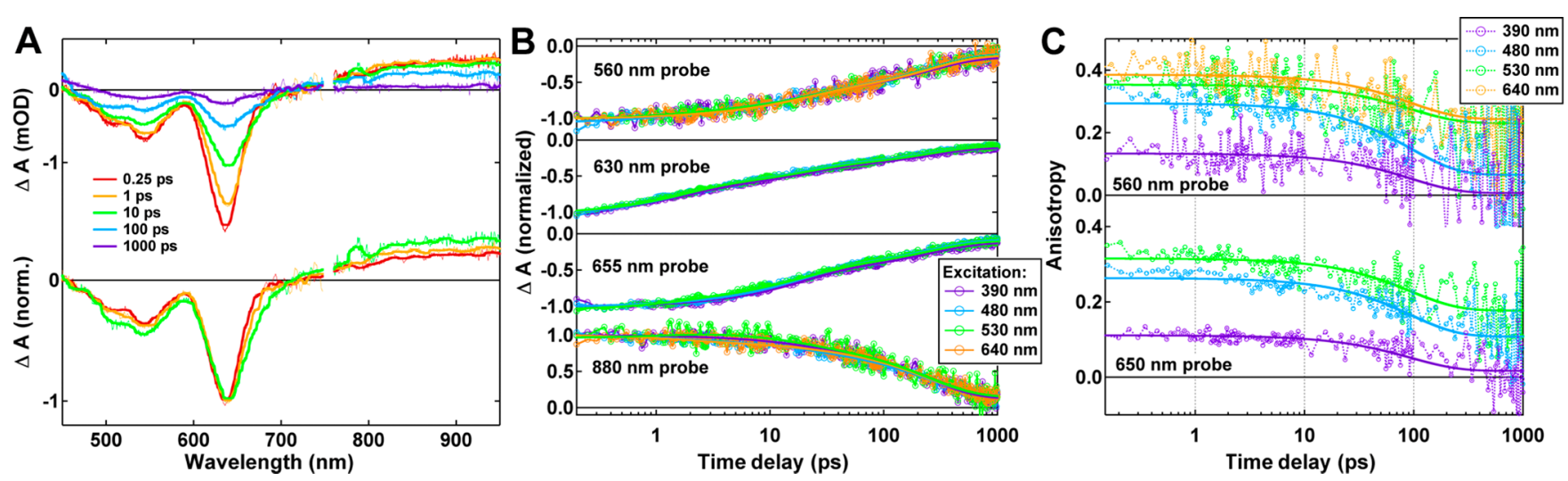

Figure 2. TA results for neat PBDTTPD film. (A) TA spectra recorded at various time delays following $390 \mathrm{~nm}$ excitation (top) and corresponding normalized TA spectra revealing spectral shifts (bottom). The smoothed thick lines are represented on top of the raw experimental data. (B) Normalized TA dynamics recorded at various spectral positions upon excitation at the four different wavelengths shown in the legend. (C) Anisotropy decay calculated from the TA dynamics at 560 and $650 \mathrm{~nm}$ with parallel and perpendicular polarization, at the different excitation wavelengths. For (B) and $(\mathrm{C})$, solid lines correspond to the best multiexponential fit. 
laser excitation always leads to some inhomogeneity in excited chromophores. Finally, the results show that neither the exciton decay nor the yield of long-lived states depends on $\lambda_{\mathrm{ex}}$.

Even if $\lambda_{\mathrm{ex}}$ negligibly influences the TA dynamics in neat PBDTTPD film, the anisotropy is strongly affected (Figure 2C, Table 1). The anisotropy was calculated from the dynamics

Table 1. Initial Anisotropy $\left(r_{0}\right)$, Anisotropy Decay Time Constant $\left(\tau_{\mathrm{r}}\right)$ and Final Anisotropy $\left(r_{\text {final }}\right)$ Obtained from the Analysis of Polarization-Sensitive TA Data for PBDTTPD and PBDTTPD:PCBM

\begin{tabular}{ccccc}
$\begin{array}{c}\lambda_{\text {probe }}(\mathrm{nm}) \\
\text { PBDTTPD }\end{array}$ & $\lambda_{\text {ex }}(\mathrm{nm})$ & $r_{0}$ & $\tau_{\mathrm{r}}(\mathrm{ps})$ & $r_{\text {final }}$ \\
560 & & & & \\
& 390 & 0.13 & 93 & 0.01 \\
& 480 & 0.29 & 93 & 0.07 \\
& 530 & 0.35 & 93 & 0.23 \\
650 & 640 & 0.39 & 93 & 0.24 \\
& 390 & 0.11 & 93 & 0.02 \\
& 480 & 0.26 & 93 & 0.11 \\
PBDTTPD:PCBM & 530 & 0.32 & 93 & 0.18 \\
560 & 390 & 0.23 & 240 & \\
& 530 & 0.40 & 240 & 0.06 \\
& 640 & 0.39 & 240 & 0.29 \\
650 & 390 & 0.09 & 240 & 0.02 \\
& 480 & 0.32 & 240 & 0.20 \\
& 530 & 0.37 & 240 & 0.29 \\
880 & 390 & 0.09 & 240 & 0.05 \\
& 640 & 0.33 & 240 & 0.23 \\
\hline
\end{tabular}

recorded with parallel and perpendicular polarization between pump and probe according to standard procedure, after verifying a perfect match between the calculated and measured magic angle data (Figure S10, SI). When the GSB signature is probed at $560 \mathrm{~nm}$, the initial anisotropy $\left(r_{0}\right)$ upon $640 \mathrm{~nm}$ excitation is close to the theoretical maximum of 0.4 , indicating parallel excited and probed transition dipole moments (indeed, absorption and GSB involve the same transition). Nevertheless, $r_{0}$ becomes smaller at shorter $\lambda_{\text {ex }}$ and is only 0.13 at $390 \mathrm{~nm}$. A similar trend is observed at the $650 \mathrm{~nm}$ probe wavelength, where both GSB and SE contribute. Decreased $r_{0}$, which becomes more pronounced at shorter $\lambda_{\mathrm{ex}}$, has been theoretically predicted and observed in TA and fluorescence experiments for several conjugated polymers. ${ }^{33,37-40}$ It is due to ultrafast relaxation processes that occur faster than the $\sim 100$ fs experimental time resolution. This can be IC, if the higher electronic states have different dipole orientations than the lowest excited state. However, the electronic transition dipoles are usually oriented along the polymer backbone. ${ }^{17}$ Therefore, it is more likely that the decrease of $r_{0}$ involves localization of an initially delocalized excited state, caused by electronic relaxation through a manifold of delocalized states formed by coupling of the chromophoric units and/or by self-trapping of the exciton due to strong coupling to nuclear modes. ${ }^{4,32,37,38}$ The former is expected to be predominant for PBDTTPD, as torsional self-trapping is limited. Higher energy excitation leads to population of higher-lying, more delocalized states, so that localization is more pronounced during relaxation, leading to greater loss of anisotropy within the time resolution and lower $r_{0}$. The relaxation through the manifold of delocalized states becomes negligible with $640 \mathrm{~nm}$ excitation near the band edge $\left(r_{0}=0.39\right)$.
Independently of pump and probe wavelength, the TA anisotropy in PBDTTPD film decays with a time constant of 93 ps to an offset value $\left(r_{\text {final }}\right)$ at long time delays when only triplets and polarons are left (Table 1). Given the limited conformational relaxation in the polymer, we assign the slow anisotropy decay to the reorientation of the probed transmission dipole moment when the exciton hops between chromophoric units. The slower time constant compared to the $1.6 / 18$ ps spectral shift in the SE, caused by a similar phenomenon, is explained by the fact that spectral dynamics probe only downhill energy transfer, while anisotropy decay is also sensitive to isoenergetic hops. The slow loss of anisotropy indicates that multistep hopping within the polymer chain and probably between neighboring chains is necessary to significantly reduce anisotropy, pointing to a rather extended chain conformation.

Electron and Hole Transfer in PBDTTPD:PCBM. For the PBDTTPD:PCBM blend, decreasing $\lambda_{\mathrm{ex}}$ leads not only to more excess energy in the system but also to enhanced absorption by the fullerene. The dotted blue absorption spectrum (neat PBDTTPD film) and dotted black spectrum (neat PCBM film) in Figure 1 are scaled so that their sum reproduces the absorption bands of the used PBDTTPD:PCBM sample. As described in detail in the SI, we used the spectral components to estimate that the relative fraction of photons absorbed by PCBM increases from $3 \%$ at $640 \mathrm{~nm}$ to $69 \%$ at $390 \mathrm{~nm}$ (Figure 1). Thus, interfacial CS in the studied PBDTTPD:PCBM thin film can in principle occur either by electron transfer (ET) from the photoexcited donor, or by hole transfer (HT) from the photoexcited acceptor, leading to population of the same (possibly "hot") charge transfer state (CT, Figure 3A), and subsequently to free charges. Both the ET and $\mathrm{HT}$ processes are energetically favorable. ${ }^{19}$

We have determined that positively charged PBDTTPD has a flat absorption in the $660-1000 \mathrm{~nm}$ region with a very characteristic peak at $880 \mathrm{~nm} .^{7}$ The signature has been independently verified by PIA spectroscopy and absorption of the oxidized polymer. ${ }^{19}$ In Figure 3B, the TA spectra at selected time delays are compared for excitation at $390 \mathrm{~nm}$ (strong PCBM absorption) and at $530 \mathrm{~nm}$ (predominant PBDTTPD absorption). Even without any further analysis, several important conclusions can be drawn from the TA spectra:

(1) At both $\lambda_{\mathrm{ex}}$, the peak at $880 \mathrm{~nm}$ is present already at the early time delay, confirming that some CS (by ET or HT) occurs promptly, as we have previously shown within the $100 \mathrm{fs}$ resolution of the experiment. ${ }^{7}$

(2) For $\lambda_{\mathrm{ex}}=530 \mathrm{~nm}$, the spectral features of the neutral polymer exciton (SE and ESA, similar as in the neat PBDTTPD film) are present at 0.5 ps. They disappear almost completely within $10 \mathrm{ps}$, with a concerted rise of the $880 \mathrm{~nm}$ charge peak, pointing to fast ET.

(3) For $\lambda_{\mathrm{ex}}=390 \mathrm{~nm}$, there is an increase of polymer GSB that accompanies the rise of the $880 \mathrm{~nm}$ polaron band, as expected if the polymer states are indirectly populated via HT from photoexcited PCBM. The HT is much slower than the ET, since it occurs predominantly between 10 and 1000 ps.

(4) At both $\lambda_{\mathrm{ex}}$, the 1000 ps signature is the same and can be assigned to charged PBDTTPD, showing that CS is complete, whether it occurred by ET or HT. Moreover, the amplitude of the 1000 ps spectrum is the same, revealing similar CS efficiency, since the photon flux was constant.

In the following, we will refer to the CS processes that are slower than the experimental time resolution as "delayed" (as opposed to "prompt"). To investigate the more delayed ET and 

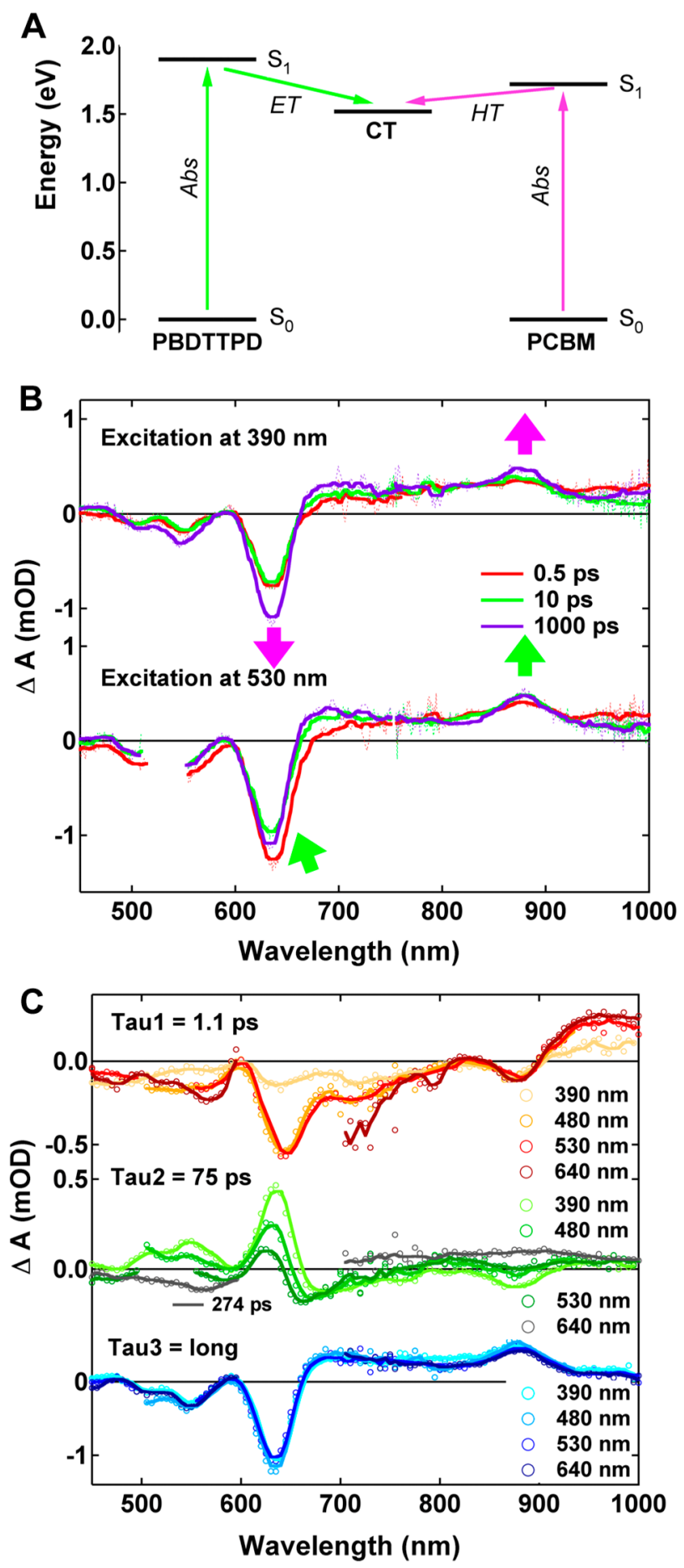

Figure 3. (A) Energy level diagram showing the first excited state of PBDTTPD and PCBM, as well as the energy of the corresponding charge transfer (CT) state (values from ref 19). Electron transfer (ET) from the photoexcited polymer and hole transfer (HT) from the photoexcited fullerene are shown as green and magenta arrows, respectively. (B) TA spectra recorded for the PBDTTPD:PCBM blend at selected time delays following 390 or $530 \mathrm{~nm}$ excitation. (C) Amplitude spectra associated with the different time constants obtained by multiexponential global analysis of the PBDTTPD:PCBM TA data recorded at the different excitation wavelengths shown in the legend. For (B) and (C), the smoothed thick lines are represented on top of the raw experimental data.
HT components, the TA dynamics at the four pump wavelengths are depicted for selected probe wavelengths in Figure 4A. They were normalized for better comparison at long times, although the necessary scaling was weak, since the dynamics converge to a similar amplitude within experimental
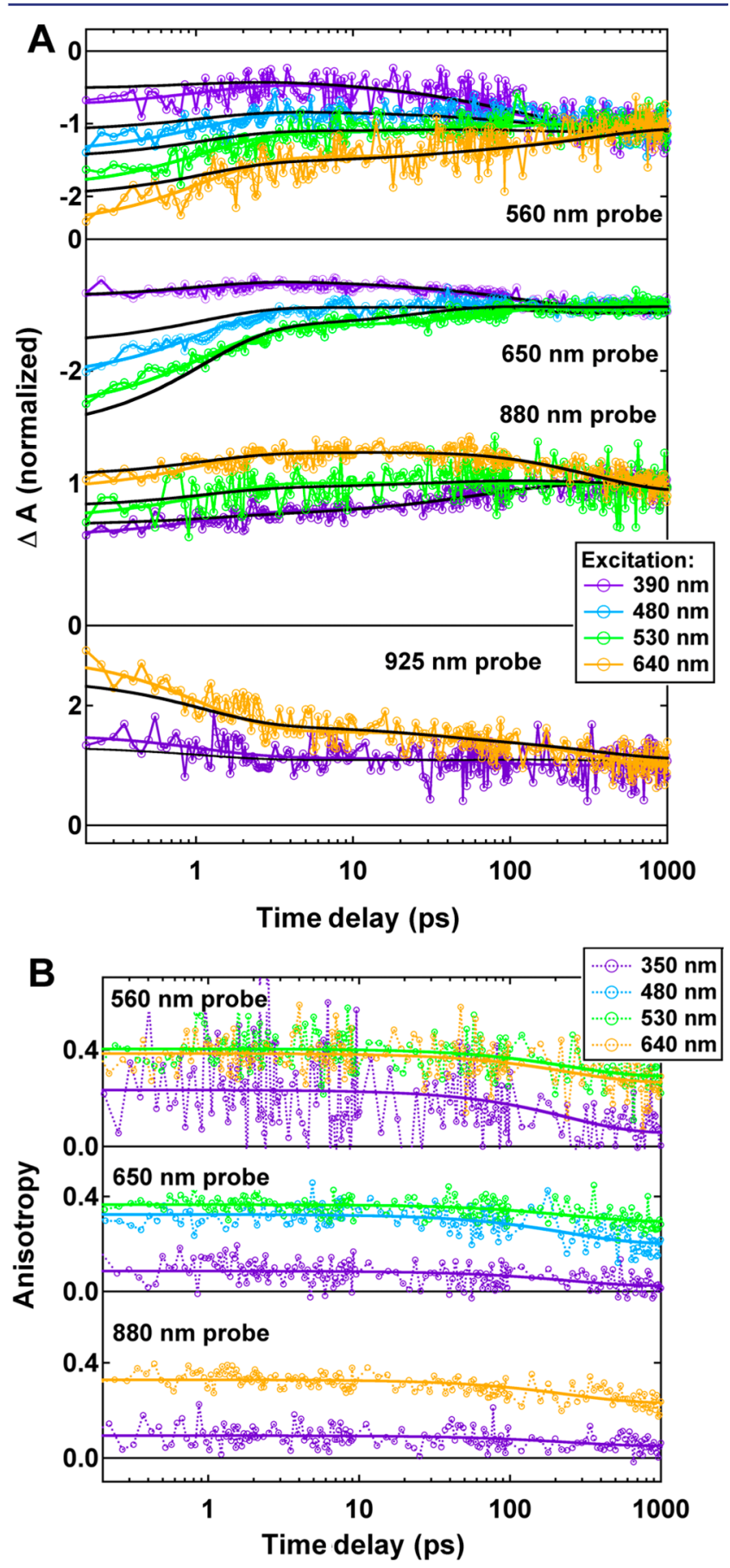

Figure 4. (A) TA dynamics for the PBDTTPD:PCBM blend, normalized at long time delays, recorded at various spectral positions upon excitation at the four different wavelengths. The solid lines in color correspond to the best global multiexponential fit, while the solid lines in black correspond to the global fit obtained using the kinetic model described in the text. (B) Anisotropy decay calculated from the TA dynamics of PBDTTPD:PCBM probed at 560, 650, and $880 \mathrm{~nm}$ at the different excitation wavelengths. Solid lines correspond to the best multiexponential fit. 
noise (Figure S11, SI). Unlike that for neat PBDTTPD, there is clear dependence of the dynamics on $\lambda_{\mathrm{ex}}$ in the blend. We note a fast component on the $\sim 1$ ps time scale, which becomes predominant at the longer $\lambda_{\text {ex }}$ and which we assign to ET. It is characterized by SE decay $(650 \mathrm{~nm})$, polaron absorption rise $(880 \mathrm{~nm})$ and polymer ESA decay $(925 \mathrm{~nm})$. The dynamics associated with HT (increase of the polymer GSB at 560 and 650 $\mathrm{nm}$, rise of the polaron band at $880 \mathrm{~nm}$ ) occur on the slower $\sim 100$ ps time scale and are enhanced at short $\lambda_{\mathrm{ex}}$. There is also some decay of all spectral features within hundreds of picoseconds, particularly visible for $\lambda_{\mathrm{ex}}=640 \mathrm{~nm}$. Nevertheless, most of the TA signal remains as a long-lived plateau.

In order to obtain a more quantitative idea about the time scales of delayed ET and HT, we globally analyzed the dynamics probed every $5 \mathrm{~nm}$ throughout the TA spectra at the different $\lambda_{\mathrm{ex}}$. Apart from being more robust to noise-related artifacts encountered at a single wavelength, the global analysis allows correlating the evolution of different signatures in the TA spectrum. ${ }^{41}$ Developing a kinetic model to describe the dynamics in complex polymer:fullerene systems requires imposing certain assumptions; therefore, we started our analysis using simply multiexponential functions (details of the procedure are given in the SI). A biexponential function with 1.1 and 75 ps time constants and an offset corresponding to long-lived signals were sufficient to globally reproduce the data. To visualize at one glance the concerted evolution of the TA bands, the pre-exponential factors associated with the global time constants are represented as amplitude spectra in Figure 3C. The sign of the amplitude indicates whether the process associated with a time constant leads to a rise or decay of the TA signal at a given probe wavelength. Since we carefully kept the number of absorbed photons constant when $\lambda_{\mathrm{ex}}$ was varied, the amplitudes can be directly compared.

The shape of the amplitude spectrum associated with $\tau_{1}=1.1$ ps varies little with $\lambda_{\text {ex }}$ and can be unambiguously assigned to ET (Figure 3C). The negative SE band in the $610-780 \mathrm{~nm}$ region, close to the steady-state emission spectrum of PBDTTPD, ${ }^{7}$ clearly indicates decay of the polymer exciton. Concomitant rise of the $880 \mathrm{~nm}$ polaron peak (see negative indent in this region) is strong evidence that the quenching occurs directly by ET to the fullerene. There is also decay of the polymer ESA in the 950 $\mathrm{nm}$ region and apparent decrease of the GSB below $600 \mathrm{~nm}$, more likely caused by rise of an overlapping positive polaron band. The overall amplitude of the $\tau_{1}$ spectrum is strongly reduced at $\lambda_{\mathrm{ex}}=390 \mathrm{~nm}$ (limited polymer absorption) and enhanced at $\lambda_{\mathrm{ex}}=640 \mathrm{~nm}$. Alternatively to ET, excitation energy transfer (EET) to PCBM can quench excitons in conjugated polymers. ${ }^{19,42}$ However, the PBDTTPD exciton decay would in this case be accompanied by a rise of the PCBM exciton signatures (Figure S8), rather than by a rise of the $880 \mathrm{~nm}$ charge band as is observed here. The only way to reconcile the occurrence of EET with our data would be that it is followed by ultrafast HT, so that the overall process appears as a single step, rate limited by the EET. We cannot exclude this possibility, but will not specifically refer to it in the following, as its outcome and effect on the TA spectra are the same as for a single ET step.

The amplitude spectrum of $\tau_{2}=75$ ps obtained from the analysis of the PBDTTPD:PCBM dynamics can be predominantly assigned to HT from PCBM, since the positive band below $670 \mathrm{~nm}$ (resembling the steady-state absorption of PBDTTPD) indicates growth of the polymer GSB, while the 880 $\mathrm{nm}$ polaron peak also increases (Figure $3 \mathrm{C}$ ). The rest of the spectrum points to rise of the flat part of the polaron absorption, and decay of the PCBM ESA, which has a weak signature throughout the visible and nIR region (Figure S8, SI). ${ }^{43}$ Although we discuss HT as splitting of a PCBM exciton in the presence of PBDTTPD, we cannot exclude the alternative HT scenario of hole injection into the polymer following spontaneous charge dissociation within PCBM clusters and charge migration (this would lead to similar evolution of the TA spectra). The process has been demonstrated ${ }^{44,45}$ but is probably slower than what we observe here. ${ }^{46}$ The weight of the 75 ps component is strongly reduced at lower excitation energy and vanishes at $640 \mathrm{~nm}$, where PCBM hardly absorbs (Figure 3C). Here, a weak decay component of the GSB and polaron band with a 274 ps time constant appears due to geminate charge recombination (gCR). It is probable that some gCR also occurs at the other $\lambda_{\mathrm{ex}}$ and is mixed into the $75 \mathrm{ps}$ amplitude spectrum, since it is masked by slow rise of the charge signature due to HT (see discussion below). We also observe a negative signature in the $660 \mathrm{~nm}$ region of the $75 \mathrm{ps}$ amplitude spectrum, which increases going from 390 to $530 \mathrm{~nm}$. This is most likely due to decay of the polymer SE, caused by a weak contribution of slower ET components.

Finally, $\tau_{3}=$ "long" represents the population of charge carriers that survives beyond 1000 ps, which is substantial for the photovoltaically efficient polymer (Figure 3C). Independently of $\lambda_{\text {ex }}$ the shape of the associated spectrum is always characterized by the same polymer GSB and charge signature. A very important result is that the magnitude of this amplitude spectrum is also independent of $\lambda_{\text {ex }}$ within experimental uncertainty. Since the number of absorbed photons was kept constant, we conclude that the overall efficiency of CS is similar upon polymer or fullerene excitation, i.e. the same number of long-lived carriers is generated. Even if HT is slower than ET, it efficiently competes with any other deactivation pathways of the PCBM singlet exciton. We have investigated the excited-state dynamics of neat PCBM film (see Figure S8 and discussion in the SI) and found that the main deactivation of the PCBM exciton is intersystem crossing to the triplet state, which occurs on a much slower (hundreds of picoseconds) time scale compared to the 75 ps HT process. ${ }^{47}$ The independence of charge yield on $\lambda_{\text {ex }}$ agrees with reported internal quantum efficiency (IQE) in corresponding PBDTTPD:PCBM solar cells, which is high and constant across the spectrum. ${ }^{10}$

The Effect of Excess Energy on Charge Separation. As described above, charges are generated promptly, as well as via delayed ET and HT processes in the PBDTTPD:PCBM blend. We will now discuss whether the prompt charges are generated from higher-lying "hot" states in presence of excess energy. According to the above anisotropy data obtained with neat PBDTTPD film, higher-lying delocalized states are indeed populated in the polymer (except with excitation near the band edge at $640 \mathrm{~nm}$ ), leading to ultrafast loss of initial anisotropy (Table 1). Given the similar time scale of ultrafast relaxation through the manifold of hot states in the polymer and the prompt ET in the blend $(<100-150 \mathrm{fs})$, it is possible that the electron is transferred before the excited state of PBDTTPD has time to relax, thus from the hot delocalized states populated in presence of excess energy. ${ }^{4,5,34,48}$ We have carried out polarization-sensitive measurements on PBDTTPD:PCBM in order to verify this (Figure 4B). In the blend, $r_{0}$ at shorter $\lambda_{\mathrm{ex}}$ is expected to be lower than in the neat polymer, due to increasing excitation of PCBM and indirect population of the polymer states via HT (one molecule is excited while the other is probed without particular orientation between them). The opposite is 
observed for $\lambda_{\mathrm{ex}}=480$ and $530 \mathrm{~nm}$, where the initial anisotropy probed at 560 or $650 \mathrm{~nm}$ is always a little higher for PBDTTPD:PCBM than for neat PBDTTPD (Table 1, Figures 4B). The drop of $r_{0}$ due to fullerene excitation only becomes apparent at $\lambda_{\mathrm{ex}}=390 \mathrm{~nm}$. This result confirms that the ultrafast polarization loss due to relaxation through the hot states is prevented in the blend, because prompt ET with excess energy occurs from the higher-lying delocalized polymer states, before this relaxation.

An important question is whether the observed occurrence of hot exciton dissociation in presence of excess energy has any effect on the yield of promptly generated charges. It has been observed in other polymer:fullerene blends that ultrafast ET is enabled by the initial delocalization in the polymer excited state (before relaxation), allowing it to sample a greater spatial extent of the $\mathrm{BHJ}{ }^{4,34,48}$ Since higher-lying delocalized states in PBDTTPD cannot be reached at $\lambda_{\text {ex }}=640 \mathrm{~nm}$, one would in this case expect a reduction of the prompt process. This should on the other hand be enhanced at shorter $\lambda_{\text {ex }}$ as higher initial delocalization would allow excitons generated further from a fullerene in the $\mathrm{BHJ}$ to undergo prompt ET. To verify this hypothesis, we will now estimate the yield of prompt CS, which occurs within the $150 \mathrm{fs}$ time resolution of the experiment, at the different excitation wavelengths.

In Figure 5A, the properly scaled exciton absorption (from the neat PBDTTPD data) is subtracted from the early (150 fs) TA spectrum of PBDTTPD:PCBM recorded at $\lambda_{\mathrm{ex}}=640 \mathrm{~nm}$, where only the polymer absorbs. This yields the spectral signature of the charges with the characteristic $880 \mathrm{~nm}$ polaron band. The amplitude of the prompt charge spectrum is roughly $20 \%$ of the maximum charge absorption at $20-50 \mathrm{ps}$, when delayed ET is complete and before the signal decays due to gCR. Knowing that the 150 fs spectrum of PBDTTPD:PCBM with $640 \mathrm{~nm}$ excitation is essentially composed of signatures from the polymer exciton and promptly generated charges, we have then decomposed it into a linear combination of the two contributions (with reference spectra taken from the neat PBDTTPD spectrum at $150 \mathrm{fs}$ and from the PBDTTPD:PCBM spectrum at $1 \mathrm{~ns}$, respectively). ${ }^{49}$ At the shorter excitation wavelengths, we added the spectrum of the PCBM exciton (from neat PCBM film) as a third component in the decomposition of the early TA spectra. The outcome of the analysis for $\lambda_{\mathrm{ex}}=390$ and $640 \mathrm{~nm}$ is shown in Figure 5C, while details of the procedure and more results are given in the SI (Figure S4).

Next, the extinction coefficient spectra, linking the transient absorbance to the concentration of the three involved species (PBDTTPD excitons, PCBM excitons and charges) were determined (Figure S5, details are given in the S.I.). In brief, we assumed that the charge concentration for PBDTTPD:PCBM upon $640 \mathrm{~nm}$ excitation at 20 ps (after fast ET and before slow gCR) is equal to the absorbed photon density, yielding the extinction coefficient of the charges. The coefficients for the other species where then determined from the data with $\lambda_{\mathrm{ex}}=390$ and $640 \mathrm{~nm}$, assuming that the sum of the exciton and charge concentrations at $150 \mathrm{fs}$ (where the individual spectral components are known) is equal to the absorbed photon density. With the extinction coefficients in hand, we calculated the concentrations of the species present in the $150 \mathrm{fs}$ TA spectra, and their yield expressed as a fraction total initial concentration (Tables 2 and S3, SI). As expected, more PBDTTPD excitons are present at longer $\lambda_{\text {ex }}\left([\text { PBDTTPD }]_{\text {init }}\right)$, while more PCBM excitons are present at short $\lambda_{\text {ex }}$ $\left([\mathrm{PCBM}]_{\mathrm{init}}\right)$. More surprisingly, we found that the yield of
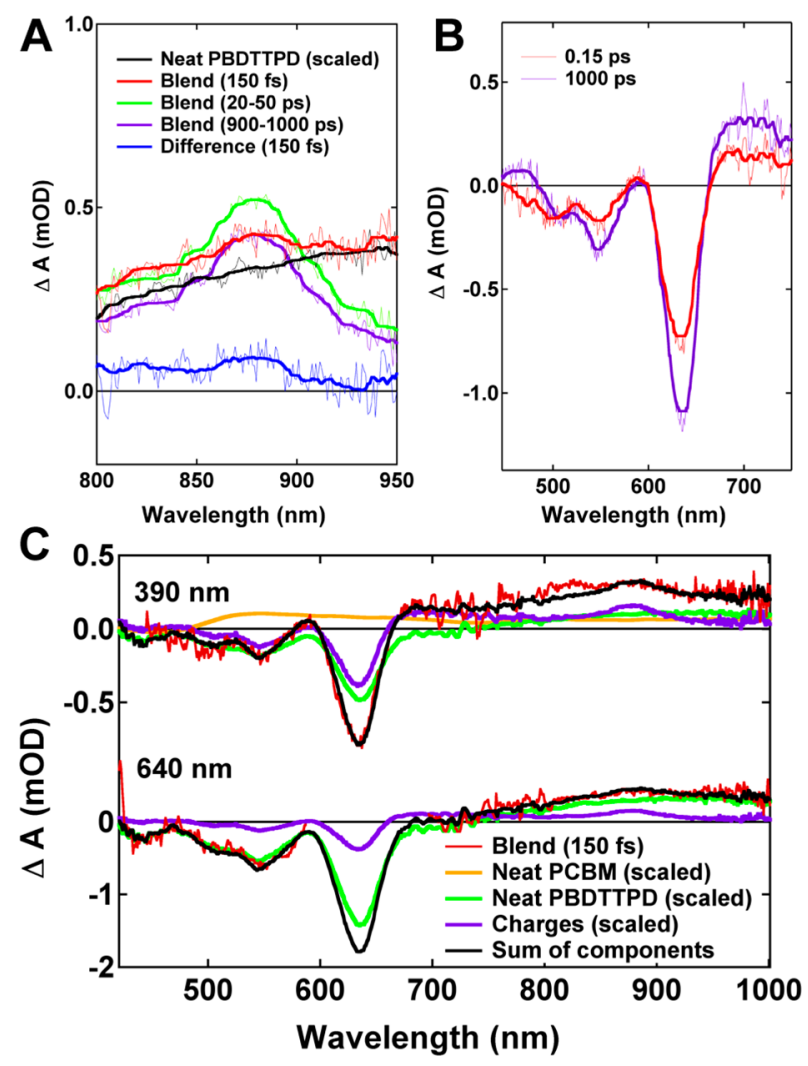

Figure 5. (A) Estimation of the prompt charge spectrum in PBDTTPD:PCBM upon $640 \mathrm{~nm}$ excitation from the difference between the TA spectrum at $150 \mathrm{fs}$ and the scaled spectrum of neat polymer at early times. Some spectra were averaged over several time delays to reduce noise and the thick lines are smoothed. (B) Estimation of the prompt hole transfer yield upon $390 \mathrm{~nm}$ excitation from the initial polymer GSB amplitude. (C) Decomposition of the early (150 fs) TA spectra of PBDTTPD:PCBM recorded at different excitation wavelengths using a linear combination of the signatures of the charge spectrum, PCBM exciton spectrum and PBDTTPD exciton spectrum.

Table 2. Contribution (Expressed as a Percentage of Total Initial Concentration) of the Different Species Present in the Early (150 fs) TA Spectra of PBDTTPD:PCBM Recorded at Different Excitation Wavelengths, and Yield of Long-Lived Charge Carriers at 1 ns

\begin{tabular}{ccccc}
$\lambda_{\text {ex }}(\mathrm{nm})$ & $\begin{array}{c}{[\text { Charge }]_{\text {init }}} \\
(\%)\end{array}$ & $\begin{array}{c}{[\mathrm{PBDTTPD}]_{\text {init }}} \\
(\%)\end{array}$ & $\begin{array}{c}{[\mathrm{PCBM}]_{\text {init }}} \\
(\%)\end{array}$ & $\begin{array}{c}{[\text { Charge }]_{\text {long }}} \\
(\%)\end{array}$ \\
390 & 28 & 25 & 47 & 84 \\
480 & 24 & 51 & 25 & 78 \\
530 & 21 & 69 & 11 & 84 \\
640 & 27 & 73 & 0 & 74 \\
\hline
\end{tabular}

promptly generated charges $\left([\text { Charge }]_{\text {init }}\right)$ is $25 \pm 3 \%$, independently of the excitation wavelength.

This result can be interpreted in two ways. First, prompt CS might occur solely by ET (no contribution from prompt HT), so that an increase of prompt ET yield in presence of excess energy compensates the decreased absorption by PBDTTPD at short $\lambda_{\mathrm{ex}}$ leading by coincidence to a constant prompt charge yield. Second, prompt CS might occur by both ET and HT with a yield that is independent of $\lambda_{\mathrm{ex}}$ and of the population of hot states. We can discard the first case, because prompt HT does occur, and in significant yield. With $\lambda_{\mathrm{ex}}=390 \mathrm{~nm}$, the amplitude of the polymer GSB at early times (100-150 fs) is already $65 \%$ of the 
maximal value at long time delays, although only $31 \%$ of photons are absorbed by the polymer at this wavelength (Figure 5B). Even admitting that the final GSB signal is reduced by up to $20 \%$ due to gCR, we can deduce that $25-35 \%$ of the polymer states are indirectly populated within the experimental time resolution via prompt HT. Thus, $25-35 \%$ represents a good estimate for the fraction of fullerene excitons undergoing prompt HT. Ultrafast HT (as fast as $30 \mathrm{fs}$ ) has also been reported for other polymer:fullerene blends. ${ }^{29}$ We therefore conclude that prompt ET and HT both occur in a yield that is independent of $\lambda_{\text {ex }}$.

At $640 \mathrm{~nm}$ (in the absence of PCBM excitation), the yield of prompt ET is $27 \%$ ([Charge $]_{\text {long }}$, Table 2$)$. By assuming a similar value for prompt ET with $390 \mathrm{~nm}$ excitation, we deduce an efficiency for prompt HT of $29 \%$ based on the contributions to the $150 \mathrm{fs}$ spectrum (see details in the SI). The excellent agreement with the value estimated above from the early GSB confirms the validity of our interpretation. By admitting a constant yield of prompt ET at all $\lambda_{\mathrm{ex}}$, we also found the fraction of photons initially absorbed by PBDTTPD and PCBM in the blend using the decomposition of the early TA spectra (Table S3, SI). There is again very good agreement with the fractions independently estimated from the steady-state absorption spectrum (Figure 1). This agreement also confirms that we were correct by assuming that only prompt CS (and no prompt EET from excited PBDTTPD to PCBM) occurs within $150 \mathrm{fs}$, which would have strongly increased the contribution of PCBM excitons in the early spectra, even without direct fullerene excitation at $640 \mathrm{~nm}$. In summary, our findings show that, although prompt ET occurs from hot states in presence of excess energy according to the anisotropy data, this does not have a significant effect on the yield of the process. The latter is equally efficient at $\lambda_{\mathrm{ex}}=640 \mathrm{~nm}$, in the absence of any hot exciton dissociation. This is possibly true for PBDTTPD:PCBM, because even the relaxed exciton in PBDTTPD is significantly delocalized in the rigid polymer. We note that short-lived delocalization and hot states likely play a more important role in polymers where self-trapping strongly localizes the exciton.

Using the extinction coefficients and total initial species concentration, we have also deduced the yield of long-lived charges at $1 \mathrm{~ns}$, which is $80 \pm 5 \%$, without any obvious trend with $\lambda_{\text {ex }}$ (Table 2). We believe that at least a part of the charges at $1 \mathrm{~ns}$ are free, as bimolecular charge recombination occurs on this time scale at high fluence, ${ }^{7}$ and as the 240 ps anisotropy decay at all probe wavelengths is possibly caused by migration of free charges (Table 1, Figure 4B). The 20\% loss of long-lived charge yield is due to gCR, which is obvious as a (fluence-independent) $\sim 300$ ps decay of the charge features at $\lambda_{\mathrm{ex}}=640 \mathrm{~nm}$ (Figures 3C and 4A). This recombination occurs for charges that have separated between PBDTTPD and PCBM, but are still Coulomb bound in a CT state across the interface. Recently, there has been lively discussion about the role of the CT state in gCR and about its possible dissociation to free charges from hot delocalized $\mathrm{CT}_{\mathrm{n}}$ states. ${ }^{4,23,24,50}$ This discussion is beyond the scope of the present manuscript, because it is in our case challenging to clearly distinguish free from bound charges for PBDTTPD:PCBM. The fact that the gCR decay is observed only for $\lambda_{\mathrm{ex}}=640 \mathrm{~nm}$ might suggest that the delocalization with near band edge excitation is not sufficient to generate free charges from the CT state. However, this is in contradiction with the wavelength-independent charge yield at $1 \mathrm{~ns}$ (Table 2) and with the reported constant IQE across the spectrum. ${ }^{10}$ Therefore, we suggest the gCR takes place at all excitation wavelengths, but is masked by the slow rise of the polaron signatures due to HT. We conclude that the yield of long-lived charges in PBDTTPD:PCBM is independent or only very weakly dependent $(<10 \%$, hidden in our experimental uncertainty) on the excess energy of photoexcitation. Our study opens interesting questions on whether gCR depends on the pathway of CS (ET or HT, prompt or delayed), ${ }^{28}$ which will we will address with more specialized techniques in a future study.

The Role of BHJ Structure. In the following, we will show that the origin of the prompt and delayed CS components in PBDTTPD:PCBM is closely related to the structure (morphology) of the $\mathrm{BHJ}$. Above, we found that about $25 \%$ of charges in PBDTTPD:PCBM appear promptly within $150 \mathrm{fs}$. It is generally not clear how prompt CS in polymer:fullerene blends can be so fast, given that excitons are unlikely to undergo significant diffusion on the $<100$ fs time scale, but still need to somehow reach a donor-acceptor interface prior to CS. ${ }^{34,51}$ We suggest here that the prompt processes in PBDTTPD:PCBM occur for excitons formed near a CS site, thus at the edge of pure phase domains or most probably in regions where PBDTTPD and PCBM are intimately mixed. Like this, exciton diffusion is no longer necessary. The observed independence of prompt CS yield on $\lambda_{\text {ex }}$ strongly supports that the process occurs in intermixed regions with fixed polymer-fullerene distance, so that increasing the initial exciton delocalization in presence of excess energy does not make a difference. The prompt charge yield $(25 \%)$ is in this case determined by the fraction of intermixed phase present in the $\mathrm{BHJ}$, independently of whether the PCBM or PBDTTPD is initially excited and whether excess energy is present. This is in excellent agreement with reported X-ray diffraction data. ${ }^{10}$ Those show that about $25 \%$ of PCBM by weight (compared to total polymer mass) mixes at the molecular level into amorphous PBDTTPD regions. A high fullerene concentration in the mixed domains is necessary to ensure percolation pathways for electron transport. ${ }^{10} \mathrm{We}$ found that about $27 \%$ of the initially formed PBDTTPD excitons undergo prompt ET, while about $29 \%$ of initially formed PCBM excitons undergo prompt HT. The significant proportion of PCBM excitons concerned by prompt CS confirms that the concentration of fullerene in the intermixed regions is high.

To further support that prompt CS is a short-range process occurring in intermixed regions, we have estimated the distance between the polymer and fullerene during ultrafast ET. We have used pump fluence dependent data to elucidate the question. The dynamics of the polymer GSB in PBDTTPD:PCBM probed at $560 \mathrm{~nm}$ as a function of fluence are shown in Figure 6 $\left(\lambda_{\text {ex }}=530 \mathrm{~nm}\right)$. When the signal amplitude at $1000 \mathrm{ps}$ is plotted versus fluence, it deviates strongly from linearity due to the bimolecular annihilation and recombination processes that we have discussed elsewhere. ${ }^{7}$ However, the initial magnitude of the GSB (at $0.1 \mathrm{ps)}$ ) starts deviating slightly from linearity only at 50 $\mu \mathrm{J} / \mathrm{cm}^{2}$. This deviation is evidence that annihilation of PBDTTPD by other excitons or charges can be ultrafast $(<100 \mathrm{fs})$, if the concentration of the interacting species is high enough at important fluence. We assume that annihilation occurring on such a short time scale is a static quenching process, i.e. that no diffusion is necessary because the species are photogenerated at a reacting distance. Using the absorbance at the excitation wavelength and corresponding estimated film thickness $(\sim 90 \mathrm{~nm}),{ }^{52}$ we calculate an excitation density of $7.8 \times$ $10^{18}$ photons $/ \mathrm{cm}^{3}$ at $50 \mu \mathrm{J} / \mathrm{cm}^{2}$. With $\lambda_{\mathrm{ex}}=530 \mathrm{~nm}, 81 \%$ of the photons are absorbed by the polymer (Figure 1), which takes up approximately $1 / 3$ of the $\mathrm{BHJ}$ volume (1:2 PBDTTPB:PCBM 

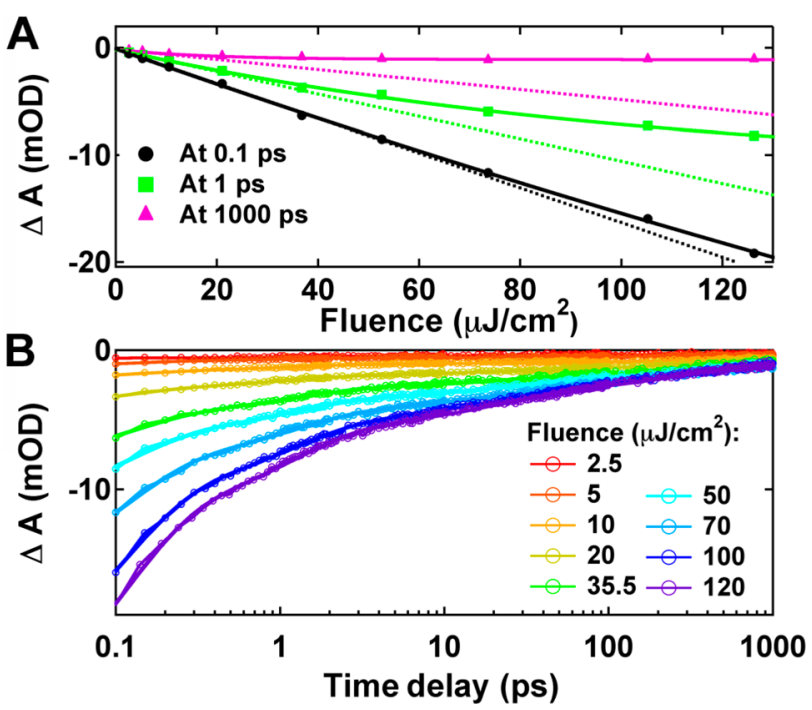

Figure 6. (A) TA intensity at several time delays of the PBDTTPD GSB, probed at $560 \mathrm{~nm}$ for PBDTTPD:PCBM, as a function of $530 \mathrm{~nm}$ pump fluence. (B) Corresponding GSB dynamics.

weight ratio, assuming comparable densities). ${ }^{53}$ The excitation density in the polymer $\left(d_{0}\right)$ is thus roughly $1.9 \times 10^{19}$ photons/ $\mathrm{cm}^{3}$, meaning that the excitons are $\sim 2.3 \mathrm{~nm}$ apart $\left(\left(3 / 4 \pi d_{0}\right)^{1 / 3}\right.$, for spherical volume) at the onset of ultrafast annihilation.

Below this fluence, an exciton in the polymer does not undergo annihilation on the $<100 \mathrm{fs}$ time scale, but we saw that prompt ET does occur. In simplified terms, this is because excitons in intermixed regions are closer to a fullerene than to another excited annihilation partner. Both ultrafast annihilation and prompt ET occur with a similar rate $(<100 \mathrm{fs})$ and are static quenching processes involving excitons directly generated at a reacting distance. We can therefore reasonably use the $\sim 2 \mathrm{~nm}$ annihilation distance as a rough estimate for how close the excited polymer is to PCBM during prompt ET. This reasoning presumes similar distance dependence for the two processes. While annihilation is typically described in terms of resonant energy transfer (RET), the point-dipole approximation used to estimate the Förster radius from spectral overlap breaks down for delocalized excitons in conjugated polymers. ${ }^{54-57} \mathrm{We}$ therefore assume that the reaction distance for prompt ET and annihilation is roughly similar, and is approximately given by the size of the delocalized exciton. Indeed, $\sim 2 \mathrm{~nm}$ seems a plausible size for a moderately delocalized exciton in a planar polymer such as PBDTTPD.

The analysis of the early TA spectra has shown that the majority (75\%) of excitons in PBDTTPD:PCBM undergo delayed rather than prompt $\mathrm{CS}$, which is surprising for such a highly efficient solar cell material. The delayed ET is in fact still very fast given the predominant associated 1.1 ps time constant (Figure 3C). Judging from 99.7\% luminescence quenching in PBDTTPD:PCBM, ${ }^{18}$ the delayed quenching pathway is highly efficient and leads to quasi quantitative generation of charges. It most probably occurs if photons are absorbed into relatively small pure polymer domains, so that exciton diffusion becomes necessary prior to ET. The existence of pure PBDTTPD aggregates is again confirmed by the X-ray diffraction study. ${ }^{10}$ Diffusion in conjugated polymers occurs by multistep intrachain and interchain exciton hopping between chromophoric segments. We have seen for neat PBDTTPD that this a highly multiphasic process, which usually requires a description by complex models based on random walk..$^{36,58,59}$ Nevertheless, we find an exponential 1.1 ps time constant for delayed ET, pointing to more monophasic behavior. This can be rationalized by considering that the rate of the process is limited by a single exciton hopping step, while the intrinsic ET step is ultrafast (as evidenced by its prompt occurrence in intermixed regions). Indeed, a single exciton hopping step can be exponential and typically occurs on a time scale of $0.5-1 \mathrm{ps}^{34,36,60}$

In order to get a rough idea of the distance of exciton diffusion related with the 1.1 ps ET process, we followed the same reasoning as for prompt ET. We previously noticed that the delayed ET process in PBDTTPD:PCBM starts competing with exciton-exciton and exciton-charge annihilation around 8-10 $\mu \mathrm{J} / \mathrm{cm}^{2}$ (see also deviation of the 1 ps GSB signal vs fluence from linearity around $12 \mu \mathrm{J} / \mathrm{cm}^{2}$, Figure $\left.6 \mathrm{~A}\right){ }^{7}$ Assuming that both annihilation and ET are intrinsically ultrafast (since we observed them within $<100 \mathrm{fs}$ ), and that both are rate limited on the $1 \mathrm{ps}$ time scale by diffusion to a comparable reacting distance given by exciton size, we estimate a PBDTTPD exciton distance from PCBM of $\sim 4 \mathrm{~nm}$ for delayed ET. This suggests a diameter of about $8 \mathrm{~nm}$ for the pure PBDTTPD domains. We note that the exciton diffusion coefficient $\left(D_{\text {diff }}\right)$ implied by one-dimensional migration over $4 \mathrm{~nm}$ within $1 \mathrm{ps}\left(\sim 1.6 \times 10^{-1} \mathrm{~cm}^{2} / \mathrm{s}\right)$ is 2 orders of magnitude larger than the one of $2-3 \times 10^{-3} \mathrm{~cm}^{2} / \mathrm{s}$ typically reported for conjugated polymers. ${ }^{55,61}$ This discrepancy arises because the exciton is not a point but is delocalized over a few nanometers along the conjugated backbone, so that it can sample a larger spatial extent. ${ }^{9}$ Using the $\sim 2 \mathrm{~nm}$ distance that we obtained for ultrafast annihilation as an estimate of the PBDTTPD exciton size, only one exciton hop is necessary to cover $\sim 4 \mathrm{~nm}$. This is in perfect agreement with the exponential ET rate as discussed above.

Depending on the inter- and intrachain coupling between chromophore units, exciton hopping can either be faster within the chain or between chains. ${ }^{34,58,62,63}$ For PBDTTPD, exciton migration on the short $\sim 4 \mathrm{~nm}$ scale to a CS site most probably occurs through the polymer chain, since we found elsewhere that the 1.1 ps ET process is not affected when the molecular packing of PBDTTPD is deteriorated by using different side-chain derivatives of the polymer. ${ }^{64}$ There is a weak contribution of even slower ET components mixed with the 75 ps time constant of delayed HT (Figure 3C). We assign this to excitons that cannot reach a CS site with a single intrachain hop, so that slower diffusion (multiple hopping steps, interchain) is necessary. The slow ET components are enhanced for shorter PBDTTPD chains, which cannot span the pure domains. ${ }^{64}$

We have shown that a large fraction of PCBM excitons undergo relatively slow $\mathrm{HT}$ on the $\sim 100$ ps time scale, which still leads to near quantitative generation of charges (in similar yield as ET). We stipulate that those fullerene excitons are generated in pure regions of clustered PCBM, as observed by X-ray diffraction. ${ }^{10}$ The driving force for HT in PBDTTPD:PCBM is favorable $(0.2 \mathrm{eV}),{ }^{19}$ so that it is probably slow diffusion through the fullerene domain to an interface that limits the rate of the process. This has already been suggested for a PCBM blend with another low bandgap polymer, where equally slow $\mathrm{HT}$ was observed. ${ }^{6}$ Indeed, the exciton diffusion coefficient of $\mathrm{C}_{60}$ is 7.5 $\times 10^{-4} \mathrm{~cm}^{2} / \mathrm{s}$, ${ }^{65}$ thus smaller than the one of $2-3 \times 10^{-3} \mathrm{~cm}^{2} / \mathrm{s}$ in conjugated polymers. ${ }^{55,61}$ Using this coefficient, we estimate that the PCBM exciton diffuses by $\sim 2.5 \mathrm{~nm}$ in $75 \mathrm{ps}$ (the time constant found for HT by multiexponential global analysis). This is most likely underestimated, since delocalization between coupled PCBM molecules can increase the exciton size. ${ }^{45}$ Thus, 
we obtain a lower limit of $5 \mathrm{~nm}$ for the diameter of pure PCBM domains in PBDTTPD:PCBM. Given the $0.7-0.8 \mathrm{~nm}$ size of a $\mathrm{C}_{60}$ molecule, ${ }^{66}$ excitons formed within those domains need to cover about 3 molecules to reach an interface prior to HT. The fact that we could reproduce the HT process with an exponential 75 ps time constant supports that the PCBM clusters are small, so that there is a weak distribution of diffusion distances. We had to use several exponentials to describe HT in another polymer:fullerene blend with large PCBM domains. ${ }^{64}$ The link of the prompt and delayed CS dynamics to a three-phase $\mathrm{BHJ}$ morphology (with intermixed regions, PBDTTPD aggregates and PCBM clusters) is summarized schematically in Figure 7. We are currently investigating PBDTTPD:PCBM blends with varying fullerene contents to further verify this picture.

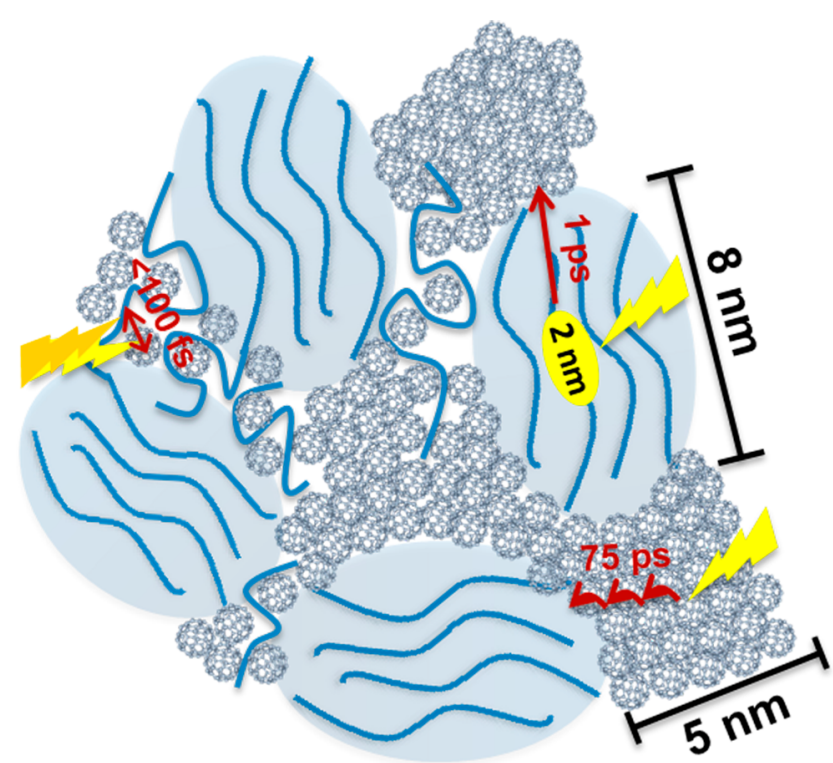

Figure 7. Schematic representation of the proposed three-phase morphology in PBDTTPD:PCBM, together with related charge separation dynamics by electron and hole transfer.

Overall Kinetic Model. As a final verification of the mechanisms that we have proposed here for the generation of charges in PBDTTPD:PCBM, we have formulated them into a kinetic model. The latter was used to globally analyze the TA dynamics at the four excitation wavelengths, and at probe wavelengths taken every $5 \mathrm{~nm}$ throughout the TA spectra (see details in the SI). The TA signal amplitude $(S(t))$ is given by the sum of contributions from the excitons and the charges, where $\varepsilon$ is the extinction coefficient (previously determined, Figure S5, $\mathrm{SI}), \mathrm{l}$ is the film thickness and []$_{\text {tot }}$ is the total (time-dependent) species concentration:

$$
\begin{aligned}
& S(t)=\varepsilon_{\mathrm{PBDTTPD}} \cdot 1 \cdot[\mathrm{PBDTTPD}]_{\mathrm{tot}}+\varepsilon_{\mathrm{PCBM}} \cdot 1 \cdot[\mathrm{PCBM}]_{\mathrm{tot}} \\
& \quad+\varepsilon_{\text {charges }} \cdot 1 \cdot[\text { Charge }]_{\mathrm{tot}}
\end{aligned}
$$

We assume that the concentration of PBDTTPD excitons decays to zero due to prompt and delayed ET. We have seen that the majority of delayed ET occurs with an exponential time constant of $1.1 \mathrm{ps}$, which could be confirmed using the kinetic model ( $\tau_{\mathrm{ET} 1}$, Table 3$)$. We have added an additional exponential decay term $\left(\tau_{\mathrm{ET} 2}=27.8 \mathrm{ps}, 20 \%\right.$, Table 3$)$ to include the weak contribution of slower ET components that we observed in the preliminary multiexponential analysis. A description based on
Table 3. Parameters Obtained from the Global Analysis of the PBDTTPD:PCBM TA Dynamics Using the Kinetic Model Described in the Text

\begin{tabular}{cccccccc}
$\begin{array}{c}\lambda_{\mathrm{ex}} \\
(\mathrm{nm})\end{array}$ & $a_{1}$ & $\begin{array}{c}\tau_{\mathrm{ET} 1} \\
(\mathrm{ps})\end{array}$ & $\begin{array}{c}\tau_{\mathrm{ET} 2} \\
(\mathrm{ps})\end{array}$ & $\begin{array}{c}\tau_{\mathrm{HT}} \\
(\mathrm{ps})\end{array}$ & $\beta_{\mathrm{HT}}$ & $\begin{array}{c}\tau_{\mathrm{gCR}} \\
(\mathrm{ps})\end{array}$ & $x_{\mathrm{gCR}}$ \\
390 & 0.8 & 1.1 & 27.8 & 134.3 & 0.72 & 332.6 & 0.17 \\
480 & 0.8 & 1.1 & 27.8 & 134.3 & 0.72 & 332.6 & 0.14 \\
530 & 0.8 & 1.1 & 27.8 & 134.3 & 0.72 & 332.6 & 0.09 \\
640 & 0.8 & 1.1 & 27.8 & 134.3 & 0.72 & 332.6 & 0.25 \\
\hline
\end{tabular}

random walk exciton diffusion and thus accounting for multiphasic quenching of excitons that have to undergo several, possibly interchain, hopping steps prior to CS, might be more appropriate. $^{36,58,59}$ This would however lead to an unreasonable number of parameters in our kinetic model, for a process that is weak and temporally mixed with HT. Therefore we have limited the analysis to the use of the second exponential, knowing that a distribution of time constants can be analyzed by the sum of exponentials. $^{67}$ The total concentration of PBDTTPD excitons is thus given by the below equation, where $[\mathrm{PBDTTPD}]_{\text {init }}$ is the concentration at $150 \mathrm{fs}$, so that excitons quenched by prompt ET are accounted for.

$$
\begin{aligned}
& {\left[\mathrm{PBDTTPD}_{\mathrm{tot}}=[\mathrm{PBDTTPD}]_{\mathrm{init}}\right.} \\
& \qquad\left\{a_{1} \exp \left(-\frac{t}{\tau_{\mathrm{ET} 1}}\right)+\left(1-a_{1}\right) \exp \left(-\frac{t}{\tau_{\mathrm{ET} 2}}\right)\right\}
\end{aligned}
$$

Similarly, we assume that the concentration of PCBM excitons completely decays due to prompt and delayed HT. Occurring on the $\sim 100$ ps time scale, delayed HT is much slower than ET. It could be reproduced with a 75 ps exponential time constant during the multiexponential analysis of the TA data (Figure 3C), pointing to a weak distribution of exciton distances from a CS site (see discussion above). In order to allow for some deviation from monophasic behavior, we have stretched the exponential term in our kinetic model (equation below) ${ }^{68-70}$ This yields a characteristic time constant of $\tau_{\mathrm{HT}}=134$ ps (Table 3), in agreement with delayed HT on the $\sim 100$ ps time scale. In simple terms, the factor $\beta_{\mathrm{ET}}$ indicates how much the dynamics deviates from exponentially (for an exponential function, $\beta=1$ ). We find a value of 0.72 , thus relatively close to 1 , in support of our interpretation that diffusion-limited HT is only weakly multiphasic for the small PCBM clusters.

$$
[\mathrm{PCBM}]_{\mathrm{tot}}=[\mathrm{PCBM}]_{\mathrm{init}} \cdot \exp \left(-\left(\frac{t}{\tau_{\mathrm{HT}}}\right)^{\beta_{\mathrm{HT}}}\right)
$$

Finally, the concentration of charges increases due to prompt and delayed CS (each quenched PBDTTPD and PCBM exciton leads to a charge pair), and a small fraction of charges $\left(x_{\mathrm{gCR}}\right)$ decays due to gCR. We assume a quantitative conversion of all excitons to charges, since no other decay pathways are obvious in the TA data. The gCR thus occurs from the total population of the charges (equal to the initial total concentration $[\mathrm{Sum}]_{\text {init }}$ ), on a time scale slower than their generation (we found an exponential time constant of $\tau_{\mathrm{gCR}}=333$ ps using the kinetic model, Table 3 ). The kinetic analysis reveals a significant fraction of gCR at all excitation wavelengths (Table 3), which confirms that the process takes place not only at $\lambda_{\mathrm{ex}}=640 \mathrm{~nm}$, but also at the shorter $\lambda_{\mathrm{ex}}$, where it is masked by slower CS components. 


$$
\begin{aligned}
& {\left[_{\text {Charge }]_{\text {tot }}=[\text { Charge }]_{\text {init }}+[\mathrm{PBDTTPD}]_{\mathrm{init}}}\right.} \\
& \quad\left\{1-\left(a_{1} \exp \left(-\frac{t}{\tau_{\mathrm{ET} 1}}\right)+\left(1-a_{1}\right) \exp \left(-\frac{t}{\tau_{\mathrm{ET} 2}}\right)\right)\right\} \\
& +[\mathrm{PCBM}]_{\mathrm{init}} \cdot\left\{1-\exp \left(-\left(\frac{t}{\tau_{\mathrm{HT}}}\right)^{\beta_{\mathrm{HT}}}\right)\right\} \\
& -x_{\mathrm{gCR}} \cdot[\text { Sum }]_{\text {init }} \cdot\left\{1-\exp \left(-\frac{t}{\tau_{\mathrm{gCR}}}\right)\right\}
\end{aligned}
$$

In order to avoid an unreasonable number of free parameters, we fixed the extinction coefficients and initial species concentrations to the values previously determined (Figure S5, Table S3, SI). Thus, mainly the time constants were globally deduced with the kinetic model (Table 3). The fits to the dynamics are shown in Figure 4A (black lines) and Figure S6, SI for all probe wavelengths. Generally, the model reproduces the data very well, with some discrepancies before 1 ps (which are exaggerated in the logarithmic plots), because early spectral shift due to relaxation are not accounted for. The applicability of the kinetic model to the experimental TA data strongly supports the validity of the assumptions that we made in our interpretation of a system as complex as a polymer:fullerene blend.

\section{CONCLUSION}

In conclusion, we show that there are several pathways for lightinduced charge separation in PBDTTPD:PCBM, closely linked to the bulk heterojunction structure of the blend. Those include electron transfer from photoexcited polymer, hole transfer from photoexcited PCBM, prompt $(<100 \mathrm{fs})$ charge generation in intimately mixed polymer:fullerene regions (which can occur from nonrelaxed hot states), as well as slower electron and hole transfer from excitons formed in pure PBDTTPD or PCBM domains (diffusion to an interface is necessary). Nevertheless, the near quantitative yield of charge separation is independent of the generation pathway (electron or hole transfer, prompt or delayed, excess energy or not).

The favorable properties of the PBDTTPD:PCBM blend in terms of morphology and exciton delocalization explain this robustness of charge formation to different circumstances, and strongly contribute to the high photovoltaic performance. The three-phase morphology predicted here for PBDTTPD:PCBM (and confirmed independently by X-ray diffraction), ${ }^{10}$ has been observed for several other conjugated polymers and corresponds to the bulk heterojunction structure yielding best solar cell efficiency. ${ }^{71-73}$ The presence of the pure regions provides a driving force for charges out of the intermixed phase and pathways for efficient charge transport. ${ }^{71}$ We find that the pure domains are predominant in PBDTTPD:PCBM, so that the majority of charges is generated by delayed electron and hole transfer. The polymer aggregates and fullerene clusters are small enough $(5-8 \mathrm{~nm})$ to ensure that all excitons generated within the pure regions can reach an interface during their lifetime. An additional asset of PBDTTPD is the planarity of the polymer, allowing exciton delocalization over $\sim 2 \mathrm{~nm}$, favoring their fast diffusion out of pure domains in a single exciton hopping step. This delocalization of even the relaxed PBDTTPD exciton also explains why excess energy (enhancing short-lived delocalization) is not a necessary prerequisite for efficient charge generation.

\section{ASSOCIATED CONTENT}

\section{S Supporting Information}

Experimental methods, additional figures, and detailed explanations of the data analysis, as mentioned in the text. This material is available free of charge via the Internet at http://pubs.acs.org.

\section{AUTHOR INFORMATION}

\section{Corresponding Author}

natalie.banerij@epfl.ch

Notes

The authors declare no competing financial interest.

\section{ACKNOWLEDGMENTS}

We thank Professor Mario Leclerc for providing PBDTTPD and Prof. Jacques Moser for putting the experimental facilities at our disposal. N.B. thanks the Swiss National Science Foundation for funding through the Ambizione Fellowship PZ00P2_136853.

\section{REFERENCES}

(1) Cabanetos, C.; El, L. A.; Bartelt, J. A.; Douglas, J. D.; Mateker, W. R.; Fréchet, J. M. J.; McGehee, M. D.; Beaujuge, P. M. J. Am. Chem. Soc. 2013, 135, 4656.

(2) Zhang, M.; Gu, Y.; Guo, X.; Liu, F.; Zhang, S.; Huo, L.; Russell, T. P.; Hou, J. Adv. Mater. 2013, 25, 4944.

(3) He, Z. C.; Zhong, C. M.; Su, S. J.; Xu, M.; Wu, H. B.; Cao, Y. Nat. Photonics 2012, 6, 591.

(4) Banerji, N. J. Mater. Chem. C 2013, 1, 3052.

(5) Grancini, G.; Maiuri, M.; Fazzi, D.; Petrozza, A.; Egelhaaf, H. J.; Brida, D.; Cerullo, G.; Lanzani, G. Nat. Mater. 2013, 12, 29.

(6) Etzold, F.; Howard, I. A.; Forler, N.; Cho, D. M.; Meister, M.; Mangold, H.; Shu, J.; Hansen, M. R.; Muellen, K.; Laquai, F. J. Am. Chem. Soc. 2012, 134, 10569.

(7) Paraecattil, A. A.; Beaupré, S.; Leclerc, M.; Moser, J.-E.; Banerji, N. J. Phys. Chem. Lett. 2012, 3, 2952.

(8) Howard, I. A.; Mauer, R; Meister, M.; Laquai, F. J. Am. Chem. Soc. 2010, 132, 14866

(9) Marsh, R. A.; Hodgkiss, J. M.; Albert-Seifried, S.; Friend, R. H. Nano Lett. 2010, 10, 923.

(10) Bartelt, J. A.; Beiley, Z. M.; Hoke, E. T.; Mateker, W. R.; Douglas, J. D.; Collins, B. A.; Tumbleston, J. R.; Graham, K. R.; Amassian, A.; Ade, H.; Fréchet, J. M. J.; Toney, M. F.; McGehee, M. D. Adv. Energy Mater. 2013, 3, 364.

(11) Qu, B.; Tian, D.; Cong, Z.; Wang, W.; An, Z.; Gao, C.; Gao, Z.; Yang, H.; Zhang, L.; Xiao, L.; Chen, Z.; Gong, Q. J. Phys. Chem. C 2013, $117,3272$.

(12) Piliego, C.; Holcombe, T. W.; Douglas, J. D.; Woo, C. H.; Beaujuge, P. M.; Fréchet, J. M. J. J. Am. Chem. Soc. 2010, 132, 7595.

(13) Kim, H. P.; bin, M. Y. A. R.; Jang, J. Sol. Energy Mater. Sol. Cells 2013, 110, 87.

(14) Shuttle, C. G.; Treat, N. D.; Douglas, J. D.; Fréchet, J. M. J.; Chabinyc, M. L. Adv. Energy Mater. 2012, 2, 111.

(15) Mateker, W. R.; Douglas, J. D.; Cabanetos, C.; Sachs-Quintana, I. T.; Bartelt, J. A.; Hoke, E. T.; El, L. A.; Beaujuge, P. M.; Fréchet, J. M. J.; McGehee, M. D. Energy Environ. Sci. 2013, 6, 2529.

(16) Liu, X.; He, R.; Shen, W.; Li, M. J. Mol. Model. 2013, 19, 4283.

(17) Risko, C.; McGehee, M. D.; Bredas, J. L. Chem. Sci. 2011, 2, 1200.

(18) Kang, T. E.; Cho, H.-H.; Cho, C.-H.; Kim, K.-H.; Kang, H.; Lee, M.; Lee, S.; Kim, B.; Im, C.; Kim, B. J. ACS Appl. Mater. Interfaces 2013, $5,1$.

(19) Hoke, E. T.; Vandewal, K.; Bartelt, J. A.; Mateker, W. R.; Douglas, J. D.; Noriega, R.; Graham, K. R.; Fréchet, J. M. J.; Salleo, A.; McGehee, M. D. Adv. Energy Mater. 2013, 3, 220.

(20) Hwang, I.; Beaupre, S.; Leclerc, M.; Scholes, G. D. Chem. Sci. 2012, 3, 2270 .

(21) Fazzi, D.; Grancini, G.; Maiuri, M.; Brida, D.; Cerullo, G.; Lanzani, G. Phys. Chem. Chem. Phys. 2012, 14, 6367. 
(22) Guo, J. M.; Ohkita, H.; Benten, H.; Ito, S. J. Am. Chem. Soc. 2010, 132,6154 .

(23) Dimitrov, S. D.; Bakulin, A. A.; Nielsen, C. B.; Schroeder, B. C.; Du, J.; Bronstein, H.; McCulloch, I.; Friend, R. H.; Durrant, J. R. J. Am. Chem. Soc. 2012, 134, 18189.

(24) Bakulin, A. A.; Rao, A.; Pavelyev, V. G.; van Loosdrecht, P. H. M.; Pshenichnikov, M. S.; Niedzialek, D.; Cornil, J.; Beljonne, D.; Friend, R. H. Science 2012, 335, 1340.

(25) van der Hofstad, T. G. J.; Di Nuzzo, D.; van den Berg, M.; Janssen, R. A. J.; Meskers, S. C. J. Adv. Energy Mater. 2012, 2, 1095.

(26) Fang, Y.; Pandey, A. K.; Nardes, A. M.; Kopidakis, N.; Burn, P. L.; Meredith, P. Adv. Energy Mater. 2013, 3, 54.

(27) Dimitrov, S. D.; Nielsen, C. B.; Shoaee, S.; Shakya, T. P.; Du, J.; McCulloch, I.; Durrant, J. R. J. Phys. Chem. Lett. 2012, 3, 140.

(28) Bakulin, A. A.; Dimitrov, S. D.; Rao, A.; Chow, P. C. Y.; Nielsen, C. B.; Schroeder, B. C.; McCulloch, I.; Bakker, H. J.; Durrant, J. R.; Friend, R. H. J. Phys. Chem. Lett. 2012, 4, 209.

(29) Bakulin, A. A.; Hummelen, J. C.; Pshenichnikov, M. S.; van Loosdrecht, P. H. M. Adv. Funct. Mater. 2010, 20, 1653.

(30) Cook, S.; Katoh, R.; Furube, A. J. Phys. Chem. C 2009, 113, 2547. (31) Wienk, M. M.; Kroon, J. M.; Verhees, W. J. H.; Knol, J.; Hummelen, J. C.; van Hal, P. A.; Janssen, R. A. J. Angew. Chem., Int. Ed. 2003, 42, 3371.

(32) Hwang, I.; Scholes, G. D. Chem. Mater. 2011, 23, 610.

(33) Banerji, N.; Cowan, S.; Vauthey, E.; Heeger, A. J. J. Phys. Chem. C 2011, 115, 9726.

(34) Banerji, N.; Cowan, S.; Leclerc, M.; Vauthey, E.; Heeger, A. J. J. Am. Chem. Soc. 2010, 132, 17459.

(35) Wells, N. P.; Boudouris, B. W.; Hillmyer, M. A.; Blank, D. A. J. Phys. Chem. C 2007, 111, 15404.

(36) Sperling, J.; Milota, F.; Tortschanoff, A.; Warmuth, C.; Mollay, B.; Bassler, H.; Kauffmann, H. F. J. Chem. Phys. 2002, 117, 10877.

(37) Tozer, O. R.; Barford, W. J. Phys. Chem. A 2012, 116, 10310.

(38) Barford, W.; Boczarow, I.; Wharram, T. J. Phys. Chem. A 2011, 115,9111

(39) Guo, J. M.; Ohkita, H.; Benten, H.; Ito, S. J. Am. Chem. Soc. 2009, $131,16869$.

(40) Dykstra, T. E.; Hennebicq, E.; Beljonne, D.; Gierschner, J.; Claudio, G.; Bittner, E. R.; Knoester, J.; Scholes, G. D. J. Phys. Chem. B 2009, 113, 656.

(41) van Stokkum, I. H. M.; Larsen, D. S.; van Grondelle, R. Biochim. Biophys. Acta, Bioenerg. 2004, 1657, 82.

(42) Ward, A. J.; Ruseckas, A.; Samuel, I. D. W. J. Phys. Chem. C 2012, 116, 23931.

(43) Banerji, N.; Wang, M. F.; Fan, J.; Chesnut, E. S.; Wudl, F.; Moser, J. E. J. Mater. Chem. 2012, 22, 13286.

(44) Burkhard, G. F.; Hoke, E. T.; Beiley, Z. M.; McGehee, M. D. J. Phys. Chem. C 2012, 116, 26674.

(45) Cook, S.; Ohkita, H.; Kim, Y.; Benson-Smith, J. J.; Bradley, D. D. C.; Durrant, J. R. Chem. Phys. Lett. 2007, 445, 276.

(46) Barbour, L. W.; Hegadorn, M.; Asbury, J. B. J. Am. Chem. Soc. 2007, 129, 15884

(47) Benson-Smith, J. J.; Ohkita, H.; Cook, S.; Durrant, J. R.; Bradley, D. D. C.; Nelson, J. Dalton Trans. 2009, 10000.

(48) Kaake, L. G.; Jasieniak, J. J.; Bakus, R. C.; Welch, G. C.; Moses, D.; Bazan, G. C.; Heeger, A. J. J. Am. Chem. Soc. 2012, 134, 19828.

(49) ale - UV-Vis-IR Spectral Software, 1.2 (FluorTools, http://www. fluortools.com).

(50) Vandewal, K.; Albrecht, S.; Hoke, E. T.; Graham, K. R.; Widmer, J.; Douglas, J. D.; Schubert, M.; Mateker, W. R.; Bloking, J. T.; Burkhard, G. F.; Sellinger, A.; Fréchet, J. M. J.; Amassian, A.; Riede, M. K.; McGehee, M. D.; Neher, D.; Salleo, A. Nat. Mater. 2014, 13, 63.

(51) Brabec, C. J.; Zerza, G.; Cerullo, G.; De Silvestri, S.; Luzzati, S.; Hummelen, J. C.; Sariciftci, S. Chem. Phys. Lett. 2001, 340, 232.

(52) Aich, B. R.; Lu, J.; Beaupre, S.; Leclerc, M.; Tao, Y. Org. Electron. 2012, 13, 1736 .

(53) Treat, N. D.; Brady, M. A.; Smith, G.; Toney, M. F.; Kramer, E. J.; Hawker, C. J.; Chabinyc, M. L. Adv. Energy Mater. 2011, 1, 82.
(54) Hodgkiss, J. M.; Albert-Seifried, S.; Rao, A.; Barker, A. J.; Campbell, A. R.; Marsh, R. A.; Friend, R. H. Adv. Funct. Mater. 2012, 22, 1567.

(55) Lewis, A. J.; Ruseckas, A.; Gaudin, O. P. M.; Webster, G. R.; Burn, P. L.; Samuel, I. D. W. Org. Electron. 2006, 7, 452.

(56) Stevens, M. A.; Silva, C.; Russell, D. M.; Friend, R. H. Phys. Rev. B 2001, 63, 165213.

(57) Ward, A. J.; Ruseckas, A.; Samuel, I. D. W. J. Phys. Chem. C 2012, 116, 23931.

(58) Markov, D. E.; Blom, P. W. M. Phys. Rev. B 2006, 74, 085206.

(59) Meskers, S. C. J.; Hubner, J.; Oestreich, M.; Bassler, H. J. Phys. Chem. B 2001, 105, 9139.

(60) Bassler, H.; Brandl, V.; Deussen, M.; Gobel, E. O.; Kersting, R.; Kurz, H.; Lemmer, U.; Mahrt, R. F.; Ochse, A. Pure Appl. Chem. 1995, 67, 377.

(61) Shaw, P. E.; Ruseckas, A.; Samuel, I. D. W. Adv. Mater. 2008, 20, 3516.

(62) Nguyen, T.-Q.; Wu, J.; Doan, V.; Schwartz, B. J.; Tolbert, S. H. Science 2000, 288, 652.

(63) Watanabe, A.; Kodaira, T.; Ito, O. Chem. Phys. Lett. 1997, 273, 227.

(64) Scarongella, M.; Paraecattil, A. A.; Buchaca-Domingo, E.; Douglas, J. D.; Beaupré, S.; McCarthy-Ward, T.; Heeney, M.; Moser, J.-E.; Leclerc, M.; Fréchet, J. M. J.; Stingelin, N.; Banerji, N. J. Mater. Chem. A 2014, Submitted.

(65) Fravventura, M. C.; Hwang, J.; Suijkerbuijk, J. W. A.; Erk, P.; Siebbeles, L. D. A.; Savenije, T. J. J. Phys. Chem. Lett. 2012, 3, 2367.

(66) Goel, A.; Howard, J. B.; Vander Sande, J. B. Carbon 2004, 42, 1907.

(67) Siemiarczuk, A.; Wagner, B. D.; Ware, W. R. J. Phys. Chem. 1990, 94, 1661

(68) Berberan-Santos, M. N.; Bodunov, E. N.; Valeur, B. Chem. Phys. 2005, 315, 171.

(69) Berberan-Santos, M. N.; Valeur, B. J. Lumin. 2007, 126, 263.

(70) Johnston, D. C. Phys. Rev. B 2006, 74, 184430.

(71) Jamieson, F. C.; Domingo, E. B.; McCarthy-Ward, T.; Heeney, M.; Stingelin, N.; Durrant, J. R. Chem. Sci. 2012, 3, 485.

(72) Collins, B. A.; Tumbleston, J. R.; Ade, H. J. Phys. Chem. Lett. 2011, 2, 3135

(73) Mayer, A. C.; Toney, M. F.; Scully, S. R.; Rivnay, J.; Brabec, C. J.; Scharber, M.; Koppe, M.; Heeney, M.; McCulloch, I.; McGehee, M. D. Adv. Funct. Mater. 2009, 19, 1173. 